(C) 2018 IEEE. This is the authors version of the work. It is posted here for your personal use. Not for redistribution. The definitive Version of Record was published in Proceedings of the 2018 Design, Automation \& Test in Europe Conference \& Exhibition (DATE), Dresden, 2018, pp. 431-436.

Personal use of this material is permitted. Permission from IEEE must be obtained for all other uses, in any current or future media, including reprinting / republishing this material for advertising or promotional purposes, creating new collective works, for resale or redistribution to servers or lists, or reuse of any copyrighted component of this work in other works.

DOI: $10.23919 /$ DATE.2018.8342048 


\title{
SOH-Aware Active Cell Balancing Strategy For High Power Battery Packs
}

\author{
Alma Pröbstl, Sangyoung Park, Swaminathan Narayanaswamy, Sebastian Steinhorst, Samarjit Chakraborty \\ Technische Universität München, Germany \\ \{alma.proebstl, sangyoung.park, swaminathan.narayanaswamy, sebastian.steinhorst, samarjit.chakraborty\}@ tum.de
}

\begin{abstract}
Short drive range due to limited battery capacity and high battery depreciation costs persist to be the main deterrents to the wide adoption of Electric Vehicles (EVs). High power battery packs consisting of a large number of battery cells require extensive management, such as State of Charge (SOC) balancing and thermal management, in order to keep the operating conditions within a safe and efficient range. In this paper, we propose a novel State of Health $(\mathrm{SOH})$-aware active cell balancing technique, which is capable of extending the cycle life of the whole battery pack. In contrast to the state-of-the-art active cell balancing techniques, the proposed technique reduces the load current of cells with low SOH using the active cell balancing architecture. Based on the observation that assigning the smallest possible load current to cells with lower SOH extends cycle life, the technique identifies the most beneficial charge transfers. We find that with our proposed scheme, aging could be mitigated by up to $23.5 \%$ over passive cell balancing and $17.6 \%$ over active SOC cell balancing.
\end{abstract}

\section{INTRODUCTION}

Electric Vehicles (EVs) are seen as one of the promising alternatives to combustion engine vehicles as they have very low cost per mile and environmental impacts. The marketshare of EVs is still small, but signs of a transition towards a fully electrified powertrain are evident. Despite the optimistic forecasts, major shortcomings of EVs persist, such as i) the range anxiety due to limited battery capacity and ii) high battery depreciation costs due to State of Health ( $\mathrm{SOH}$ ) degradation. Even though battery prices are expected to drop in the near future, either manufacturers or EV owners bear the costs for a battery which will eventually become unusable within the EV and therefore be disposed due to capacity loss. This is undesirable from both the economic and the environmental perspective. If implemented, our method can help in saving several thousands of dollars.

Typically, Lithium-ion (Li-ion) cells are preferred for such high power applications due to their high energy and power densities compared to other rechargeable battery chemistries. As shown in Fig. 1, a high power battery pack is formed by connecting multiple Li-ion cells in parallel to increase the capacity and connecting these parallel cell modules in series for achieving the required high operating voltage. In order to ensure safe and efficient operation, Li-ion cells demand a tight control over their operating conditions. Operation outside their defined set of safe thresholds will reduce their cycle life and probably damage them, causing fire or explosion due to thermal runaway.

The capacity fading rate of Li-ion cells depends on various factors, such as operating temperature, average State of Charge (SOC) and SOC swing and it is generally known that high average SOC, high temperature, and large SOC swing are detrimental for aging [1]. While the $\mathrm{SOH}$ of each cell degrades, the usable capacity of the series-connected cells is determined by the cell with the lowest SOH. With repeated charge/discharge cycles, the $\mathrm{SOH}$ of all cells tends

With the support of the Technische Universität München - Institute for Advanced Study, funded by the German Excellence Initiative and the European Union Seventh Framework Programme under grant agreement no. 291763. And with the support of the German Federal Ministry for Education and Research with grant agreement no. 01DQ17018 (FutureTrans).

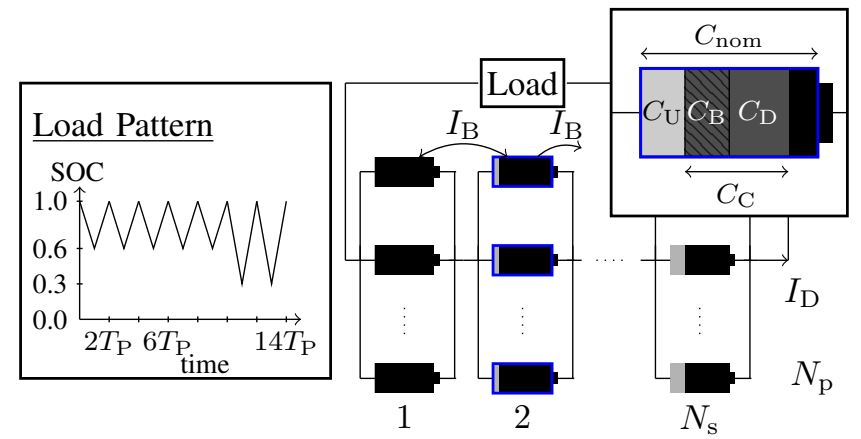

Fig. 1: A battery pack consisting of $N_{\mathrm{p}}$ parallel electrically indistinguishable cells and $N_{\mathrm{S}}$ series connected cells varying in $\mathrm{SOH} . C_{\mathrm{U}}$ is the unusable capacity due to capacity fading. The required charge is $C_{\mathrm{D}}=I_{\mathrm{D}} T_{\mathrm{P}}$. Using an active cell balancing architecture, the amount of charge transferred is $C_{\mathrm{B}}=$ $I_{\mathrm{B}} T_{\mathrm{P}}$. The effective charge transferred between cells is $C_{\mathrm{C}}$.

to diverge due to manufacturing inhomogeneities and varying temperature distribution. This also contributes to an early disposal of the battery pack from the vehicle, since current regulations demand replacement of the entire EV battery pack if any cell in the pack reaches $70 \%$ of its $\mathrm{SOH}$ value.

Conventional balancing approaches are passive, where the excess charge of cells with higher SOC is dissipated as heat across a resistor, resulting in a reduced energy efficiency. By contrast, active cell balancing approaches increase the energy output of the battery pack by transferring the excess charge between cells instead of dissipating it as heat. However, existing active cell balancing approaches only focus on equalizing the SOC at all times and inherently partially contribute towards increased cycle life [2]. By optimizing the load current assignment, the cycle life can be significantly improved further.

In this paper, we focus on the fact that the EV batteries are not always used to their full capacity in every drive cycle, and hence, it is not necessary to keep SOC equalized at all times. For instance, a typical usage profile of an EV is shown in Fig. 1] where the pack follows a driving pattern that consists of five small commuting trips during weekdays followed by two longer trips on the weekends. It is not mandatory to always maintain an equal SOC among all cells when the entire battery capacity will not be used within a single cycle. Existing methods of remaining range estimation [3] help to estimate the available SOC margin. Estimating the required charge to reach a predefined destination is crucial to enable autonomous driving and further advances are expected to be made in this domain. We propose to leverage this headroom for mitigating battery $\mathrm{SOH}$ degradation using a state-of-the-art active cell balancing architecture [4] by reducing the load current of the less healthy cells.

In particular, we propose the following contributions.

- Active cell balancing reduces stress on less healthy cells and extends cycle life. We show that this effect can be further exploited and cycle life is significantly extended (Sec. III).

- We propose a novel SOH-aware active cell balancing 
technique that adapts load currents to further mitigate aging (Secs. IV and V).

- Compared to passive and conventional active balancing, experimental results show that our technique improves cycle life by $23.5 \%$ and $17.6 \%$, respectively (Sec. VI).

\section{RELATED WORK}

SOH degradation mitigation in EVs: There are various approaches to mitigate the $\mathrm{SOH}$ degradation of $\mathrm{EV}$ batteries. An often used method for aging reduction is the charge pattern. A cost and SOH-degradation optimized charge pattern can be achieved by charging slowly, and charging shortly before the trip starts, which reduces temperature rise, and lowers the average SOC, respectively [5]. Instead of the charging pattern, the authors of [6] control the EV's heating, ventilation and air conditioning system to extend battery runtime and cycle life. In addition, battery pack reconfiguration techniques can also be used to improve the $\mathrm{SOH}$. The work in [7] proposes to connect cells with similar SOH levels in order to increase available capacity in one cycle. However, such reconfigurable systems have significant efficiency limitations due to the onresistance of reconfiguration switches. The work in [2] shows that compared to passive balancing, active SOC balancing narrows the capacity distribution among cells on the long term and increases cycle life. Our work overlays on this finding by proposing a methodology to even further increase this effect. Active cell balancing: A comprehensive overview of different cell balancing architectures is provided in [8]. Typically, the existing active cell balancing approaches are classified into capacitor-based, inductor-based and transformer-based, depending upon the type of energy storage element used for charge transfer [9]. Among them the inductor-based approaches are more preferable since they provide a higher energy efficiency compared to the capacitor-based architectures and also occupy a smaller installation volume when compared to the transformer-based counterparts. In addition to the electrical architectures, several equalization strategies are proposed in the literature that determine optimal charge transfer direction between the cells to equalize the SOC of the battery pack. For instance, four different request-driven equalization strategies are proposed in [10]. However, existing works in the domain of active cell balancing only focus on equalizing the SOC of all cells in the pack and do not consider $\mathrm{SOH}$, which we do in this paper for the first time.

Online SOH measurement: The proposed SOH-aware cell balancing technique requires an online $\mathrm{SOH}$ measurement that can be implemented in the Battery Management System (BMS). An overview of existing methods is provided in [11]. Coulomb counting is a relatively simple online $\mathrm{SOH}$ estimation method, which integrates the amount of charge flowing in and out of the battery pack. However, it does not consider the temperature effects and the self-discharge current of the battery pack and therefore does not provide accurate results. On the other hand, electrochemical impedance spectroscopy techniques that directly measure the battery internal impedance are more accurate. However, due to their requirement of high accuracy measurements and complex signal processing tasks, they are typically performed offline in a laboratory. Recently, the work of [12] suggests a real-time electrochemical impedance spectroscopy technique, paving the way for high accuracy SOH estimation at the BMS level. $\mathrm{SOH}$ estimation is continuously improving. However, $\mathrm{SOH}$ estimation techniques are not in the scope of this paper. Note that our proposed $\mathrm{SOH}$-aware balancing methodology can be

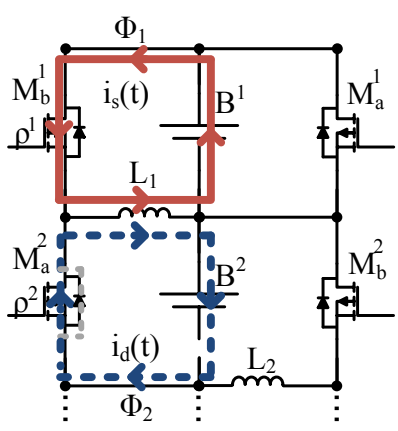

(a)

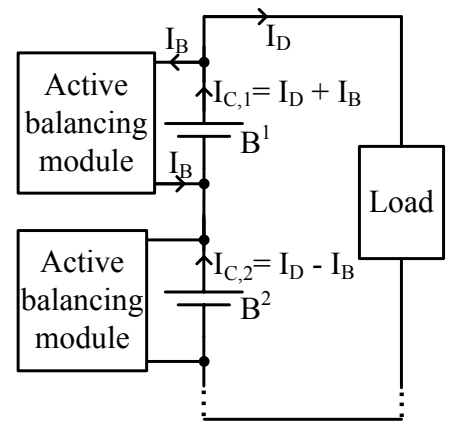

(b)
Fig. 2: (a) Operating principle of an inductor-based active cell balancing architecture proposed in [13]. (b) A higher current $\left(I_{\mathrm{D}}+I_{\mathrm{B}}\right)$ is discharged from healthier cell $B^{1}$ whereas cell $B^{2}$ sees a reduced current $\left(I_{\mathrm{D}}-I_{\mathrm{B}}\right)$.

implemented in conjunction with any of the existing $\mathrm{SOH}$ estimation techniques in the literature.

\section{Observations For ACtive Cell Balancing}

In this section, we first explain the operating principle of a state-of-the-art inductor-based active cell balancing architecture, upon which the proposed technique is built. Then, we provide some key observations, which form the groundwork for the strategies introduced in this paper.

Operating principle: A state-of-the-art inductor-based active cell balancing architecture, as proposed in [13], is shown in Fig. 2 a. Each cell is associated with a balancing module that consists of two power Metal-oxide-semiconductor field-effect transistor (MOSFET) switches $\left(M_{a}^{i}\right.$ and $M_{b}^{i}$ ) and an energy storage element, inductor $L_{i}$. Charge transfer between cells takes places in two phases, charging $\left(\Phi_{1}\right)$ and discharging $\left(\Phi_{2}\right)$ that are controlled by two high frequency control signals $\rho^{1}$ and $\rho^{2}$, respectively. During the charging phase $\Phi_{1}$, MOSFET $M_{b}^{1}$ is actuated with $\rho^{1}$ and the excess charge in cell $B^{1}$ is stored in inductor $L_{1}$. During the discharging phase, $M_{b}^{1}$ is turned $O F F$ and $M_{a}^{2}$ is actuated with $\rho^{2}$ transferring the stored energy in the inductor $L_{1}$ to cell $B^{2}$. Short free-wheeling phases during which the inductor current flows through the body-diode of the respective power MOSFETs are necessary to avoid short-circuit conditions between the cells. The balancing current ranges from 0 to a maximum value $I_{B}$ and is modified by adjusting the length of the PWM signal generated with $\rho^{1}$ and $\rho^{2}$.

Observations with two cells: We provide the key observations using a simple two-cell example. Two cells are discharged by a discharge current $I_{\mathrm{D}}$. The balancing current $I_{\mathrm{B}}$, which is limited by hardware component constraints and costs, can now either increase or decrease the load current experienced by individual cells. When one cell $B^{1}$ transfers charge to another cell $B^{2}$ through $I_{\mathrm{B}}, B^{1}$ effectively has a higher load current $I_{\mathrm{C}, 1}=I_{\mathrm{D}}+I_{\mathrm{B}}$ and $B^{2}$, which receives the charge, has effectively a lower load current $I_{\mathrm{C}, 2}=I_{\mathrm{D}}-I_{\mathrm{B}}$ as shown in Fig. 2. In this section, we ignore the transfer efficiency $\gamma$ for the sake of simplicity. However, it will be considered in Sec. V.

Observation 1: Battery aging depends on the average SOC and also the SOC swing. Avoiding high SOC helps to mitigate battery aging. Lower SOC swing means less severe capacity fading [14], [15].

Observation 2: Cells in a pack age at different speeds with temperature being the main contributor to the aging speed 


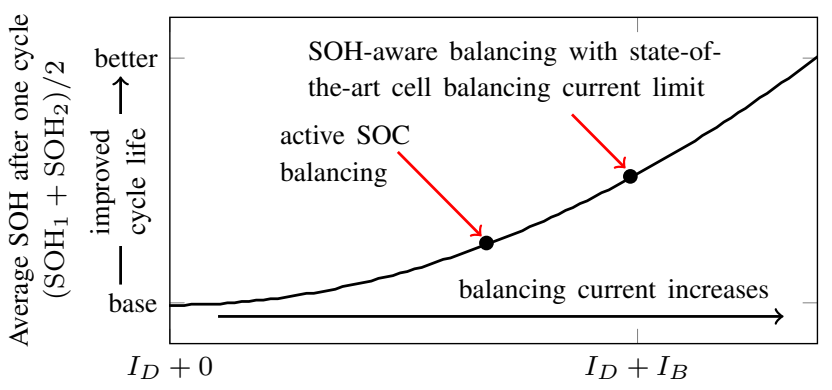

Cell current of healthier cell $I_{\mathrm{C}, 1}$

Fig. 3: Average SOH of two series connected cells after one charge/discharge cycle. Transferring charge from the healthier cell to the less healthy cell is beneficial on the long term and increases cycle life beyond SOC balancing.

divergence [14]. In high power battery packs, cooling is usually not homogeneous. Especially in automotive applications, where the pack volume needs to be minimized, there is a trade-off between achievable temperature homogeneity and the overhead introduced by the cooling system hardware. Even the most efficient state-of-the-art cooling approaches still expose the batteries to temperature stress when being charged or discharged at high rates [16]. Manufacturing variations, while depending on the cell size and quality of the manufacturing process, are another significant contributor to cell inhomogeneity which eventually promotes different aging behavior between cells [17], [18]. Also, the aging-speed slightly reduces with decreased SOH [14], [19]. Due to permanent equalization currents, parallel cells can be assumed to be electrically indistinguishable. While serial cells built up a variation in capacity because of manufacturing inhomogeneities and temperature variations.

Observation 3: Resting the less healthy cells mitigates the End of Life (EOL). With active cell balancing, the individual cell current, and hence the average SOC and also SOC swing, can be adjusted. The work in [2] confirms that stressing the healthier cell more than the less healthy cells reduces the SOH gap between healthiest and least healthy cell and prolongs cycle life. We observe the same behavior for two cells using the aging model from [1]. Fig. 3 illustrates the effect of obtaining increased cycle life for higher balancing currents. On the long term, this preserves the less healthy cells as the EOL is determined by the least healthy cell reaching a predefined $\mathrm{SOH}$ value. Note that the gain achieved in a single cycle is small, but in the long term, the effect significantly contributes to increased cycle life as will be shown in Sec. VI. We propose a strategy that intensifies this effect by further optimizing the load current assignment. I.e., we use the maximum available balancing current, which is only limited by component constraints of the balancing architecture.

Based on above observations, we develop an SOH-aware balancing strategy that increases the number of cycles of a battery pack until EOL in Sec. V.

\section{System Modeling}

\section{A. Battery Aging Model}

Using the correct aging model is crucial for evaluating the proposed cell balancing technique. Hence, we employ a widely-adopted SOH model, which describes electrochemical degradation processes by physical crack propagation mechanisms over cycling and time [1]. Further experimental evaluation in [2] supports the model behavior. Stress parameters are the cell temperature $T_{\mathrm{B}}$, SOC swing $\sigma$ and average SOC
$\overline{S O C}$, where the latter two along with the time interval $T_{\mathrm{m}}$ depict the charge/discharge current.

As the cycle interval $T_{\mathrm{m}}$ may start and end at arbitrary SOC states, the effective number of throughput cycles

$$
N=\int_{T_{\mathrm{m}}} \frac{|i(t)| d t}{2 Q_{\text {nom }}},
$$

needs to be calculated, where $i(t)$ is the charge or discharge current, $Q_{\text {nom }}$ is the nominal amount of charge that can be stored in the battery and $m$ denotes the m-th time the cell is discharged and recharged between arbitrary SOCs. A first degradation parameter accounts for the damage in midcentered cycles

$$
D_{1}=K_{\text {co }} N \exp \left((\sigma-1) \frac{T_{\text {ref }}+273}{K_{\text {ex }}\left(T_{\mathrm{B}}+273\right)}\right)+0.2 \frac{t_{\text {cycle }}}{t_{\text {life }}},
$$

where $K_{\text {co }}$ is a normalization coefficient for $N$ and $K_{\text {ex }}$ is a constant exponent for SOC swing. $T_{\text {ref }}$ is the reference battery temperature of $25^{\circ} \mathrm{C}$. The duration of one cycle is denoted by $t_{\text {cycle }}$ and $t_{\text {life }}$ is the shelf life at $25^{\circ} \mathrm{C}$ and $50 \%$ SOC until EOL. Adjusting to $\overline{S O C}$ is done in the second degradation parameter

$$
D_{2}=D_{1} \exp \left(4 K_{\mathrm{soc}}(\overline{S O C}-0.5)\right)\left(1-D\left(T_{\mathrm{m}-1}\right)\right),
$$

using a constant $K_{\mathrm{soc}}$ to account for the $\overline{S O C}$. Finally, the total increase in the degradation parameter $L$ is given by

$$
D\left(T_{\mathrm{m}}\right)=D_{2} \exp \left(K_{\mathrm{t}}\left(T_{\mathrm{B}}-T_{\text {ref }}\right) \frac{T_{\text {ref }}+273}{T_{\mathrm{B}}+273}\right),
$$

where $K_{\mathrm{t}}$ accounts for a doubling of the decay rate for each $10^{\circ} \mathrm{C}$ rise in temperature. Summing up the damage done by each cycle, one can derive the remaining life of the battery.

Besides the model, we define the aging-speed as degradation per cycle: $v_{\mathrm{a}}=\frac{\Delta S O H}{\text { cycle }}$. Further, we specify the unit to be points per million and cycle: $\frac{1 \times 10^{-6}}{\text { cycle }}=1$ ppmc. I.e., a cell with an aging-speed of 50 ppmc will have lost $5 \%$ of its initial capacity after 1000 cycles.

\section{B. Cell Balancing Efficiency}

We use the existing analytical model from [20], which is derived for inductor-based active cell balancing architectures, to calculate the energy efficiency of the charge transfer process. We allow direct charge transfers only between neighboring cells. The energy efficiency of the charge transfer process is calculated as

$$
\gamma=1-\frac{E_{\mathrm{diss}}}{E_{\mathrm{tx}}}
$$

where $E_{\mathrm{diss}}$ and $E_{\mathrm{tx}}$ are the energy dissipated and the energy transferred, respectively. The two major sources of energy dissipation in the cell balancing process are the conduction energy dissipation $E^{\text {cd }}$, due to the parasitic resistances of the circuit components and the switching energy dissipation $E^{\mathrm{sw}}$, due to the non-zero ON and OFF times of the MOSFET switch. The conduction energy dissipation in a single charge transfer cycle is calculated as:

$$
E_{\mathrm{cyc}}^{\mathrm{cd}}=Q_{\mathrm{tx}} \cdot V_{B^{1}}-Q_{\mathrm{rx}} \cdot V_{B^{2}},
$$

where $Q_{\mathrm{tx}}$ is the charge transferred by the source cell and $Q_{\mathrm{rx}}$ is the charge received by the destination cell in a single switching cycle. $V_{B^{1}}$ and $V_{B^{2}}$ are the voltages of the source and destination cells respectively. The energy dissipation due to the switching activity of MOSFETs in each charge transfer cycle is calculated as

$E_{\mathrm{cyc}}^{\mathrm{sw}}=\frac{1}{2} I_{\mathrm{peak}}\left\{t_{\mathrm{OFF}} \cdot V_{B^{1}}+t_{\mathrm{ON}} \cdot V_{B^{2}}\right\}+\frac{1}{2} C_{\mathrm{OSS}}\left\{V_{B^{1}}^{2}+V_{B^{2}}^{2}\right\}$ 


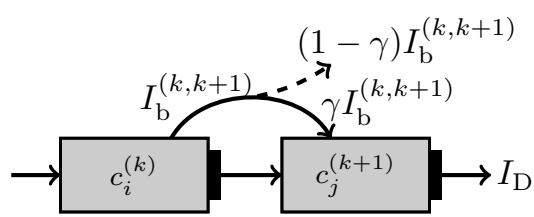

(loss)

Fig. 4: The left cell $k$ is aged less than cell $k+1$. This is shown by indices $i>j$. The efficiency of the charge transfer is denoted by $\gamma$.

where $I_{\text {peak }}$ is the final inductor current during the charging phase, $t_{\mathrm{ON}}$ and $t_{\mathrm{OFF}}$ are the turn-ON and turn-OFF times of the MOSFET switch and $C_{\mathrm{OSS}}$ is the parasitic output capacitance of the MOSFET.

However, as we propose a high-level cell balancing strategy and simulate long-term effects of batteries, it is impractical to consider circuit-level details. In the following sections, we use the average balancing current $I_{\mathrm{B}}=1 / 4 \times I_{\text {peak }}$. Also, based on our circuit-level simulations, we find that it is safe to assume a constant efficiency of $96 \%$ for typical $V_{B}$ values and balancing currents.

\section{SOH-Aware Active Cell Balancing}

The basic idea of the $\mathrm{SOH}$-aware cell balancing algorithm is to compensate the amount of charge that is discharged from cells with a lower $\mathrm{SOH}$ by transferring charge from neighboring cells with a relatively higher $\mathrm{SOH}$. We assume that the battery management system is capable of reliably measuring the capacity of cells. The transfer is done using the active cell balancing architecture from Fig. 2 We assume that the decision for the transfer direction and the amount of the cell balancing current is made at the beginning of each trip and remains fixed. This is sufficient as battery aging is a comparatively slow process. The algorithm is shown in Alg.11 We assume that the accumulated charge required $Q_{\text {req }}$ from the battery pack is known at the beginning of each trip. In a real-world implementation, this could be determined by, e.g., the destination entered in the navigation system (Alg.11. line 11. For the remainder of this paper, we consider a constant discharge current $I_{\mathrm{D}}=\frac{Q_{\mathrm{req}}}{T_{\mathrm{P}}}$ for our discussion of the algorithm (Alg.11, line2), where $T_{\mathrm{P}}$ is the duration of a trip. This is safe to assume because using average $I_{\mathrm{D}}$ is sufficient for determining $I_{\mathrm{b}}^{(k, k+1)}$ in the algorithm. However, using a time-series of $I_{D}$ values is possible without modifications.

The $S O H_{i}^{(k)}=\frac{C_{i}^{(k)}}{C_{\text {nom }}}$ of each cell is the ratio of available capacity $C_{i}^{(k)}$ and the nominal capacity $C_{\text {nom }}$ (Alg. 1 line 3 . The latter is the same for all cells. The superscript $k$ indicates the physical location of the cell within the series connection, i.e., cell $k$ is left of cell $k+1$. The subscript $i$ denotes the position of the cell within the ordered list of cell SOHs, where $C_{i}^{(m)} \leq C_{i+1}^{(n)}$ (Alg. 1 line 4 ). We assume that cells in parallel are electrically indistinguishable while cells in series vary in SOC. The discharge current of a cell $I_{\mathrm{C}}^{(k)}$ (and hence the SOC variation of cell $k$ ) depends on $I_{\mathrm{D}}$ but it is influenced by the balancing current $I_{\mathrm{b}}^{(k, k+1)}$ flowing between two cells $k$ and $k+1$. The efficiency of the transfer is denoted by $\gamma$. Fig. 4 shows a cell $k$ being discharged by $I_{\mathrm{D}} T_{\mathrm{P}}+I_{\mathrm{b}}^{(k, k+1)} T_{\mathrm{P}}$ amount of charge while cell $k+1$ receives only $\gamma I_{\mathrm{b}}^{(k, k+1)} T_{\mathrm{P}}$. The algorithm calculates this under the assumption that the voltage difference among cells are not drastic as we discussed in Sec. IV-B. Accordingly, the current seen by cell $k$ consists of $I_{\mathrm{D}}$ along with the current received by or transmitted to

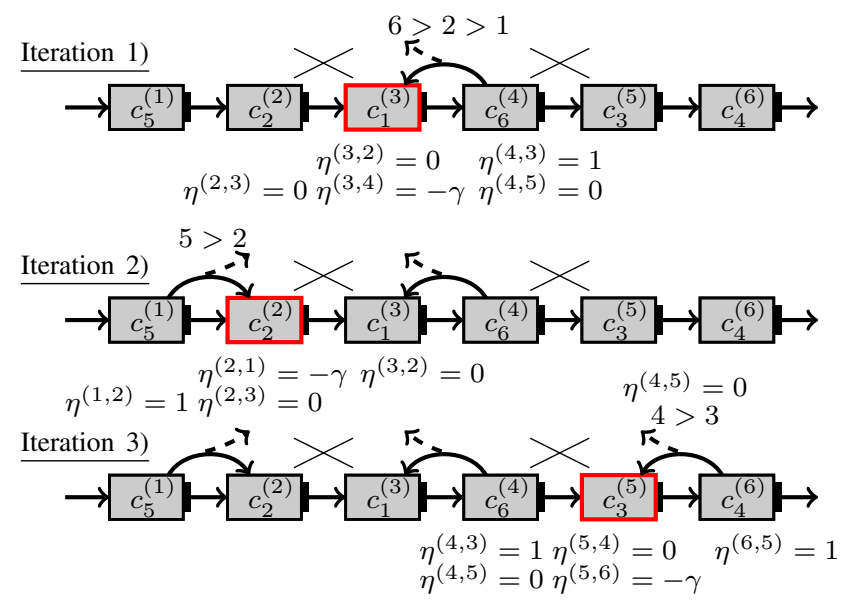

Fig. 5: Finding the concurrent charge transfers. The grouping of cells is done by iterating through the ordered health list starting with the least healthy cell and grouping it with the healthier neighboring cell.

neighboring cells:

$$
I_{\mathrm{C}}^{(k)}=I_{\mathrm{D}}+\eta^{(k, k-1)} I_{\mathrm{b}}^{(k-1, k)}+\eta^{(k, k+1)} I_{\mathrm{b}}^{(k, k+1)},
$$

where $\eta^{(m, n)} \in\{-\gamma, 0,1\}$ indicates the charge transfer direction and loss. $I_{\mathrm{b}}^{(m, n)}=I_{\mathrm{b}}^{(n, m)}$ is independent of the direction. If $\eta^{(m, n)}=1$ then $m$ transmits and $n$ receives charge. If $\eta^{(m, n)}=-\gamma$ then $m$ receives and $n$ transmits charge.

Charge transfer direction: The charge transfers between cells happen concurrently. However, a cell can only transfer charge to one neighboring cell at one time, not to both: $\eta^{(k, k-1)} \neq$ $0 \Rightarrow \eta^{(k, k+1)}=0$ and $\eta^{(k, k+1)} \neq 0 \Rightarrow \eta^{(k-1, k)}=0$. As aging is a very slow process, the $\mathrm{SOH}$ values are updated at a low rate, and transfer directions $\eta^{(k, k-1)}$ and $\eta^{(k, k+1)}$ are not adjusted during a trip but only at the beginning. The algorithm iterates through the cells sorted according to their $C_{i}^{(k)}$, denoted by subscript $i$ (health list) (Alg. 1, line 5). It marks the healthier neighbor as charge provider unless this neighbor is already grouped with another cell (Alg.1, line 6). We illustrate the rules for cell groupings in the example in Fig. 5. The formal formulation of the rules is given in Table 1 Due to space constraints, the special cases of cells $k=1$ and $k=N_{\mathrm{s}}$ are omitted, but can be easily derived. In Iteration 1), the least healthy cell $C_{1}^{(3)}$ is grouped with its right neighbor $C_{6}^{(4)}$ as this is healthier than the left neighbor $(i=6>2>1)$. Accordingly, $\eta^{(3,4)}=-\gamma$ as the cell $C_{1}^{(3)}$ receives charge and $\eta^{(4,3)}=1$ as cell $C_{6}^{(4)}$ transfers charge. Due to this grouping, cell $C_{2}^{(2)}$ cannot be grouped with cell $C_{1}^{(3)}$ and therefore, $\eta^{(3,2)}=0$ and $\eta^{(2,3)}=0$. Equally, cell $C_{6}^{(4)}$ cannot be grouped with cell $C_{3}^{(5)}$ and thus, $\eta^{(4,5)}=0$ (Table 1 line 4). In Iteration 2), a grouping partner for the second least healthy cell $C_{2}^{(2)}$ is required and the healthier neighbor $C_{5}^{(1)}$ is selected (Table] line 6). Finally in Iteration 3), the low health of cell $C_{3}^{(5)}$ should be compensated. The healthier neighbor $C_{6}^{(4)}$ is already grouped, which is indicated by $\eta^{(4,5)}=0$. Therefore, the right neighbor $C_{4}^{(6)}$, which is also healthier than cell $C_{3}^{(5)}$ is selected and the $\eta^{(m, n)}$ are updated accordingly (Table $\mathrm{I}$. line 1+5).

Balancing currents: Next the balancing currents $I_{\mathrm{b}}^{(k, k+1)}$ are calculated (Alg.1. line 9). In general, the non-zero balancing currents should be set to the maximum balancing current allowed by the architecture $I_{\mathrm{B}}$ in order to relieve the less 
TABLE I: Get the $\eta^{(m, n)}$ values depending on the already given $\eta$ values and the available charge of the two neighbors of cell $C^{(k)}: C^{(k+1)}$ and $C^{(k-1)}$. A - means that the parameter remains unchanged.

\begin{tabular}{|c|c|c|c|c|}
\hline \multirow[b]{2}{*}{ if } & \multirow{2}{*}{$C^{(k+1)}$} & \multicolumn{3}{|c|}{$\eta^{(k-1, k-2)} / \eta^{(k, k-1)} / \eta^{(k+1, k)} /$} \\
\hline & & $\eta^{(k-1, k)}$ & $\eta^{(k, k+1)}$ & $\eta^{(k+1, k+2)}$ \\
\hline 1: $\eta^{(k-1, k)}=0$ & - & $-/-$ & $0 /-$ & $-/-$ \\
\hline 2: $\quad \eta^{(k+1, k)}=0$ & - & $-/-$ & $-/ 0$ & $-1-$ \\
\hline 3: $\quad \begin{array}{l}\eta^{(k, k-1)}=\text { undef. } \\
\eta^{(k, k+1)}=\text { undef. }\end{array}$ & $>C^{(k-1)}$ & $-/ 0$ & $0 /-\gamma$ & $1 / 0$ \\
\hline 4: $\quad \begin{array}{l}\eta^{(k, k-1)}=\text { undef. } \\
\eta^{(k, k+1)}=\text { undef. }\end{array}$ & $\leq C^{(k-1)}$ & $0 / 1$ & $-\gamma / 0$ & $-/-$ \\
\hline 5: $\begin{array}{l}\eta^{(k, k-1)}=0 \\
\eta^{(k, k+1)}=\text { undef. }\end{array}$ & & $-/$ & $-1-\gamma$ & $1 /-$ \\
\hline 6: $\quad \begin{array}{l}\eta^{(k, k-1)}=0 \\
\eta^{(k, k+1)}=\text { undef. }\end{array}$ & & $-/ 1$ & $-\gamma /-$ & $-/-$ \\
\hline
\end{tabular}

TABLE II: The balancing current $I_{\mathrm{b}}^{(\nu)}$ depends on the available charge in the two neighboring cells.

\begin{tabular}{|c|c|c|c|}
\hline$I_{\mathrm{B}}$ rel. & $C_{\mathrm{h}} / T_{\mathrm{P}} \quad$ Char & $\begin{array}{l}\text { Level Check } \\
C_{1} / T_{\mathrm{P}}\end{array}$ & $I_{\mathrm{b}}^{(\nu)}$ \\
\hline 1: $I_{\mathrm{D}}>I_{\mathrm{B}}$ & $>I_{\mathrm{D}}+I_{\mathrm{B}}$ & $>I_{D}-\gamma I_{\mathrm{B}}$ & Eq.9 \\
\hline$I_{\mathrm{D}}>I_{\mathrm{B}}$ & $<I_{\mathrm{D}}+I_{\mathrm{B}}$ & $>I_{\mathrm{D}}-\gamma\left(\frac{C_{\mathrm{h}}}{T_{\mathrm{P}}}-I_{\mathrm{D}}\right)$ & Eq. 10 \\
\hline$I_{\mathrm{D}}<I_{\mathrm{B}}$ & $>I_{\mathrm{D}}\left(1+\frac{1}{\gamma}\right)$ & & Eq. 11 \\
\hline 4: $I_{\mathrm{D}}<I_{\mathrm{B}}$ & $<I_{\mathrm{D}}\left(1+\frac{1}{\gamma}\right)$ & $>I_{\mathrm{D}}-\gamma\left(\frac{C_{\mathrm{h}}}{T_{\mathrm{P}}}-I_{\mathrm{D}}\right)$ & Eq. $\overline{10}$ \\
\hline
\end{tabular}

healthy cell most as explained in Sec. III. Obs. 3).

$$
I_{\mathrm{b}}^{(\nu)}=I_{\mathrm{B}} \text {. }
$$

The variable $\nu$ is used as a placeholder for the cell pairs grouped, where $\eta^{(k, k-1)} \neq 0 \Rightarrow \nu=(k-1, k)$ and $\eta^{(k, k+1)} \neq 0 \Rightarrow \nu=(k, k+1)$. If both $\eta^{(k, k-1)}=0$ and $\eta^{(k, k+1)}=0$ then the balancing currents $I_{\mathrm{b}}^{(k-1, k)}$ and $I_{\mathrm{b}}^{(k, k+1)}$ are also zero. However, we need to consider two special cases. Firstly, if the current $I_{\mathrm{B}}$ applied over $T_{\mathrm{P}}$ would prematurely deplete the healthier cell, we need to apply a reduced current.

$$
I_{\mathrm{b}}^{(\nu)}=\frac{C_{\mathrm{h}}}{T_{\mathrm{P}}}-I_{\mathrm{D}}
$$

Secondly, if the balancing current $I_{\mathrm{B}}$ is high and the discharge current $I_{\mathrm{D}}$ is small enough, the less healthy cell could rest and

$$
I_{\mathrm{b}}^{(\nu)}=\frac{1}{\gamma} I_{\mathrm{D}}
$$

The decision criteria are depicted in Table II It takes into consideration the capacity of the healthier cell $C_{\mathrm{h}}$ and the less healthy cell $C_{\mathrm{l}}$. Assuming that the $I_{\mathrm{b}}^{(\nu)}$ are calculated while iterating through the health list ( $i$ subscripts) (Alg. 1. line 8), the following applies: $\eta^{(k, k-1)}=-\gamma \Rightarrow C_{\mathrm{h}}=C^{(k-1)}$ and $\eta^{(k, k+1)}=-\gamma \Rightarrow C_{\mathrm{h}}=C^{(k+1)}$ and $C_{\mathrm{l}}=C^{(k)}$. The case of $\eta^{(m, n)}=1$ is similar.

\section{Simulation Results}

For this paper, we compare the $\mathrm{SOH}$-aware cell balancing algorithm proposed in Section $\mathrm{V}$ with an active SOC balancing scheme and passive cell balancing. The active SOC balancing scheme equalizes only the SOC of all cells making use of the same cell balancing architecture for fair comparison [13]. We connect those neighboring cells which have the larger SOC difference. But instead of applying the maximum $I_{\mathrm{B}}$, we equalize the SOC as much as possible. If that equalization should not be possible in $T_{\mathrm{P}}$ then Eq. 10 and 11 are applied. We believe this baseline is reasonable enough, given the fact that most of the real-world battery packs utilize passive cell balancing, and active cell balancing is still a field of active research. In passive cell balancing, no balancing happens during discharge. But when charging, all cells are charged to full SOC. Cells that reach full charge earlier are discharged by
Algorithm 1 SOH-aware cell balancing algorithm. //Call this function at the start of each trip

$$
\begin{aligned}
& \text { Input: destination, } C_{i}^{(k)}, T_{\mathrm{P}} \\
& \text { Output: } I_{\mathrm{b}}^{(k, k+1)} \forall k \in 1 \ldots N_{\mathrm{s}}-1 \\
& \text { 1: } \left.Q_{\text {req }}=\text { getQreq(destination, } T_{\mathrm{P}}\right) \text {; } \\
& \text { 2: } I_{\mathrm{D}}=\operatorname{get} \operatorname{ID}\left(Q_{\text {req }}\right) \text {; } \\
& \text { 3: } S O H_{i}^{(k)}=\text { updateSOH }\left(C_{i}^{(k)}\right) \text {; } \\
& \text { 4: [healthList, } i]=\text { sortCellsAscendingSOH}\left(S O H_{i}^{(k)}\right) \text {; } \\
& \text { 5: for all cells in healthList do } \\
& \text { 6: } \quad\left[\eta^{(k-1, k-2)}, \eta^{(k-1, k)}, \eta^{(k, k-1)}, \eta^{(k, k+1)}, \eta^{(k+1, k)}\right. \\
& \text { 7: end for } \\
& \left.\eta^{(k+1, k+2)}\right]=\operatorname{getEta}\left(C_{i}^{(k)}\right) ; / / \text { Table } I \\
& \text { 8: for cell } k=1 \ldots N_{\mathrm{S}}-1 \text { do } \\
& \text { 9: }\left[I_{\mathrm{b}}^{(k, k+1)}\right]=\operatorname{getBalancingCurrent}\left(I_{\mathrm{D}}, \eta^{(k, k+1)}\right. \text {, } \\
& \left.C_{i}^{(k)}, C_{i}^{k+1}\right) \text {; // Table II }
\end{aligned}
$$$$
\text { 10: end for }
$$

resistors. This scheme has the advantage that the architecture is simple and cheap. However, its energy efficiency is low as excess charge is dissipated as heat, usable capacity of the pack is smaller, and requires that there are no outliers in cell parameters for acceptable system efficiency. However, $\mathrm{SOH}$ optimization cannot be performed with passive balancing.

We simulate a battery pack with an architecture similar to the one of the Nissan Leaf $24 \mathrm{kWh}$ battery pack which features 96 cells in series and 2 in parallel (96s2p) enhanced with the active cell balancing architecture from Sec. III. We simulate the battery pack in MATLAB. Our model includes the aging model from Sec. IV-A. The cells connected in series vary in $\mathrm{SOH}$ and aging-speed. We assume the cells' initial SOHs to be uniformly distributed in the interval $[0.9,1]$ and an agingspeed range of $[54.352,55.264]$ ppmc. This is a resemblance of the temperature inhomogeneity in a pack and the cells' manufacturing variations. If not mentioned otherwise, we assume a maximum balancing current $I_{\mathrm{B}}$ of $5 \mathrm{~A}$. We assume the period $T_{\mathrm{P}}$ to be $3600 \mathrm{~s}$. The simulation runtime of one charge-/discharge cycle is approximately $0.06 \mathrm{~s}$.

In the following, we investigate the dependency of our heuristics on (i) the balancing current, (ii) the aging-speed range and (iii) the initial aging distribution. For the first two tests, we initialize the cells with a fixed initial age distribution. The EOL is reached when the $\mathrm{SOH}$ of the least healthy cell reaches 0.7 . We compare three different user profiles: light, medium and heavy user. All three users repeat a pattern similar to the one shown in Fig. 1. For all three users, the repeated pack SOC pattern starts from $S O C_{\text {high }}=[1 ; 1 ; 1 ; 1 ; 1 ; 1 ; 1]$ and discharges to $S O C_{\text {low }}=\left[S O C_{2} ; S O C_{2} ; S O C_{2} ; S O C_{2} ; S O C_{2} ; 0.3 ; 0.3\right]$ with $S O C_{2}$ being 0.8, 0.6 and 0.3 for light, medium and heavy user, respectively. As the capacity fades, $S O C_{\text {low }}$ is adjusted. Dependency on Balancing Current: The gain of the balancing schemes strongly depends on the balancing current. For the given initial $\mathrm{SOH}$ distribution, Fig. 67 shows that for low balancing currents, the $\mathrm{SOH}$-aware cell balancing performs very similar to SOC cell balancing. For balancing currents $I_{\mathrm{B}}$ greater than $1 \mathrm{~A}, 2 \mathrm{~A}$ or $3 \mathrm{~A}$ of the heavy, medium, or light user profile, respectively, the $\mathrm{SOH}$-aware cell balancing clearly outperforms conventional SOC balancing. For approximately $I_{\mathrm{B}}=7 \mathrm{~A}$, the $\mathrm{SOH}$-aware scheme stabilizes at a gain of up to $23.5 \%$ over the passive cell balancing. The gain is similar for all profiles. The results clearly suggest that the balancing architecture is an important design decision. Investing in 


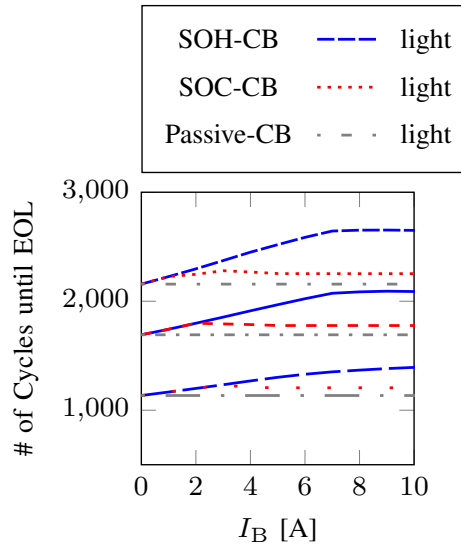

(a)
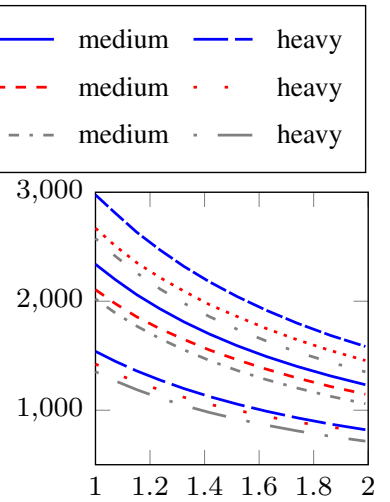

Maximum normalized aging-speed variation

(b)
Fig. 6: (a) Comparison of balancing schemes with varying balancing current $I_{\mathrm{B}}$. The aging-speed is 54.674 ppmc. (b) Comparison of balancing schemes with varying normalized aging-speed, where 1 means that all cells age at the same speed.

stronger balancing infrastructure can be rewarded by increased cycle life of the battery pack. The main difference between user profiles is the total number of cycles until EOL, where, as expected, the battery pack of the heavy user ages much faster compared to the pack of the light user.

Dependency on Aging-Speed: We investigate the impact of aging-speed variations induced by temperature inhomogeneities by increasing the interval from zero variation (all cells have an identical aging-speed) to a doubling of the agingspeed (the least healthy cell ages twice as fast as the slowest aging cell). The $\mathrm{x}$-axis in Fig. 6p shows the maximum agingspeed among a pack, normalized to a minimum aging-speed of $v_{\mathrm{a}, \min }=54.352$ ppmc. For example, the value of 1.6 denotes a normalized aging speed distribution from 1 to 1.6. The balancing current equals $5 \mathrm{~A}$. As expected, the number of cycles decreases with increasing aging-speed. Additionally, the gap between $\mathrm{SOH}$-aware and passive cell balancing is almost constant at a gain of up to $14.7 \%, 16.4 \%, 17.2 \%$ for heavy, medium and light user, respectively. The gain of SOC balancing over passive cell balancing reaches up to $11.7 \%$, $8.2 \%, 7.7 \%$ for heavy, medium and light user, respectively.

Dependency on Initial Aging Distribution: As we investigate the neighbor-only balancing techniques, the initial $\mathrm{SOH}$ distribution strongly influences the cycle life of the pack. For our example, we fix the balancing current to $5 \mathrm{~A}$ and the aging-speed $v_{\mathrm{a}}$ to $54.674 \mathrm{ppmc}$, randomly generate 20 uniform $\mathrm{SOH}$ distributions and calculate the mean cycle life. The performance in terms of cycle life of the proposed algorithm as well as passive and SOC balancing strongly depends on the initial distribution of differently aged cells within a pack. On average, the SOH-aware cell balancing outperforms the passive cell balancing by approximately $12.5 \%$ for the heavy user, $13.3 \%$ for both the medium and light user while the SOC balancing yields gains of approximately 4.4\%, 3.8\%, 3.4\% over passive cell balancing on average for heavy, medium and light user, respectively (Fig. 7). The results suggest that the arrangement of cells varying in $\mathrm{SOH}$ and aging-speed within a pack is an important criterion to be considered.

\section{CONCLUSION}

Cell balancing architectures in large battery packs equalize SOC differences between cells stemming from manufacturing variances and temperature distribution within the pack in order

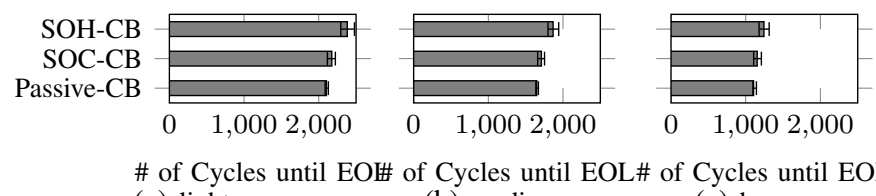
(a) light
(b) medium
(c) heavy

Fig. 7: Mean number of cycles until EOL for 20 random initial distributions of aged cells.

to increase the usable capacity within a single cycle. We show that conventional active SOC balancing algorithms do not make use of the full potential of the balancing architecture in terms of increasing cycle life. We propose a novel SOHaware balancing scheme that increases the cycle life of the battery pack by up to $23 \%$ by reducing the load current on cells with lower SOH. Considering the high prices for battery packs this leads to a massive benefit.

\section{REFERENCES}

[1] A. Millner, "Modeling lithium ion battery degradation in electric vehicles," in Proc. of CITRES, 2010.

[2] Y. Shi, K. Smith, R. Zane, and D. Anderson, "Life prediction of large lithium-ion battery packs with active and passive balancing," in Proc. of $A C C, 2017$

[3] J. Hong, S. Park, and N. Chang, "Accurate remaining range estimation for electric vehicles," in Proc. of ASP-DAC, 2016.

[4] S. Narayanaswamy, M. Kauer, S. Steinhorst, M. Lukasiewycz, and S. Chakraborty, "Modular active charge balancing for scalable battery packs," IEEE Transactions on Very Large Scale Integration (VLSI) Systems, pp. 1-14, 2016, in Press.

[5] A. Hoke, A. Brissette, K. Smith, A. Pratt, and D. Maksimovic, "Accounting for lithium-ion battery degradation in electric vehicle charging optimization," IEEE Journal of Emerging and Selected Topics in Power Electronics, vol. 2, no. 3, pp. 691-700, 2014.

[6] K. Vatanparvar and M. A. Al Faruque, "Battery lifetime-aware automotive climate control for electric vehicles," in Proc. of DAC, 2015.

[7] L. He, Y. Gu, T. Zhu, C. Liu, and K. G. Shin, "Share: Soh-aware reconfiguration to enhance deliverable capacity of large-scale battery packs," in Proc. of ICCPS, 2015.

[8] J. Cao, N. Schofield, and A. Emadi, "Battery balancing methods: A comprehensive review," in Proc. of VPPC, 2008.

[9] J. L. Gallardo, C. E. Romero, M. M. I. Milanes, and M. M. A. Guerrero, "Battery equalization active methods," Journal of Power Sources, vol. 246, pp. 934 - 949, September 2014.

[10] S. Steinhorst, M. Kauer, A. Meeuw, S. Narayanaswamy, M. Lukasiewycz, and S. Chakraborty, "Cyber-physical co-simulation framework for smart cells in scalable battery packs," ACM Transactions on Design Automation of Electronic Systems (TODAES), vol. 21, no. 4, pp. 62:1-62:26, 2016.

[11] M. Berecibar, I. Gandiaga, I. Villarreal, N. Omar, J. Van Mierlo, and P. Van den Bossche, "Critical review of state of health estimation methods of li-ion batteries for real applications," Renewable and Sustainable Energy Reviews, vol. 56, pp. 572 - 587, April 2016.

[12] E. Din, C. Schaef, K. Moffat, and J. T. Stauth, "A scalable active battery management system with embedded real-time electrochemical impedance spectroscopy," IEEE Transactions on Power Electronics, vol. 32, no. 7, pp. 5688-5698, 2017.

[13] N. H. Kutkut, "A modular nondissipative current diverter for ev battery charge equalization," in Proc. of APEC, 1998.

[14] S. F. Schuster, T. Bach, E. Fleder, J. Müller, M. Brand, G. Sextl, and A. Jossen, "Nonlinear aging characteristics of lithium-ion cells under different operational conditions," Journal of Energy Storage, vol. 1, pp. 44-53, 2015.

[15] T. Guena and P. Leblanc, "How depth of discharge affects the cycle life of lithium-metal-polymer batteries," in Proc. of INTELEC, 2006.

[16] I. A. Hunt, Y. Zhao, Y. Patel, and G. J. Offer, "Surface cooling causes accelerated degradation compared to tab cooling for lithium-ion pouch cells," Journal of The Electrochemical Society, vol. 163, no. 9, pp. A1846-A1852, 2016.

[17] M. Dubarry, N. Vuillaume, and B. Y. Liaw, "Origins and accommodation of cell variations in li-ion battery pack modeling," International Journal of Energy Research, vol. 34, no. 2, pp. 216-231, 2010.

[18] T. M. Bandhauer, S. Garimella, and T. F. Fuller, "A critical review of thermal issues in lithium-ion batteries," Journal of the Electrochemical Society, vol. 158 , no. 3, pp. 1-25, 2011.

[19] T. Reddy, Linden's Handbook of Batteries, 4th Edition. McGraw-Hill Education, 2010.

[20] M. Kauer, S. Narayanaswamy, M. Lukasiewycz, S. Steinhorst, and S. Chakraborty, "Inductor optimization for active cell balancing using geometric programming," in Proc. of DATE. IEEE, March 2015. 